\title{
Interplay between the loop-tree duality and helicity amplitudes
}

\author{
F. Driencourt-Mangin, ${ }^{1, *}$ G. Rodrigo $\oplus^{1, \dagger}$ G. F. R. Sborlini ${ }^{1,2, \ddagger}$ and W. J. Torres Bobadilla $\oplus^{1,3, \S}$ \\ ${ }^{1}$ Instituto de Física Corpuscular, Universitat de València-Consejo Superior de Investigaciones \\ Científicas, Parc Científic, E-46980 Paterna, Valencia, Spain \\ ${ }^{2}$ Deutsches Elektronen-Synchrotron DESY, Platanenallee 6, 15738 Zeuthen, Germany \\ ${ }^{3}$ Max-Planck-Institut für Physik, Werner-Heisenberg-Institut, 80805 München, Germany
}

(Received 30 March 2021; revised 2 December 2021; accepted 2 January 2022; published 12 January 2022)

\begin{abstract}
The spinor-helicity formalism has proven to be very efficient in the calculation of scattering amplitudes in quantum field theory, while the loop-tree duality (LTD) representation of multiloop integrals exhibits appealing and interesting advantages with respect to other approaches. In view of the most recent developments in LTD, we exploit the synergies with the spinor-helicity formalism to analyze illustrative one- and two-loop scattering processes. We focus our discussion on the local UV renormalization of IR and UV finite helicity amplitudes and present a fully automated numerical implementation that provides efficient expressions, which are integrable directly in four space-time dimensions.
\end{abstract}

DOI: $10.1103 /$ PhysRevD.105.016012

\section{INTRODUCTION}

In order to unveil the fundamental components of matter and their interactions, it is necessary to analyze highlyprecise experimental data obtained from colliders using accurate theoretical predictions. However, the established theoretical frameworks, i.e., the Standard Model, involves very complicated mathematical equations, whose exact solutions are unknown in many physically relevant processes. Thus, most of the computations performed nowadays rely on the perturbative approach, which naturally leads to Feynman loop amplitudes and loop integrals.

With the purpose of achieving a higher accuracy in the theoretical predictions, it is mandatory to explore higher perturbative orders and compute multiloop amplitudes with high multiplicity. To tackle these calculations, several methods have been developed in the last years. On the one hand, there has been enormous progress in the algebraic handling of scattering amplitudes in gauge theories by using alternative kinematic variables as the ones provided by the spinor-helicity formalism [1]. Also, there was an important improvement due to the study of the mathematical properties of the scattering amplitudes, for instance, in the color sector [2-4] and the development

\footnotetext{
*felix.dm@ific.uv.es

†german.rodrigo@csic.es

*german.sborlini@desy.de

torres@mpp.mpg.de
}

Published by the American Physical Society under the terms of the Creative Commons Attribution 4.0 International license. Further distribution of this work must maintain attribution to the author(s) and the published article's title, journal citation, and DOI. Funded by SCOAP ${ }^{3}$. of new regularization strategies $[5,6]$. These techniques lead to a much more efficient treatment of the scattering amplitudes, exploiting several symmetries to simplify the underlying expressions.

On the other hand, there were also great advances in the calculation of multiloop Feynman integrals, both analytically and numerically [7]. In particular, pointing towards a more efficient numerical implementation, we have been developing a novel strategy based on the loop-tree duality (LTD) theorem [9-18]. This theorem allows to decompose any loop amplitude (or loop integral) as the sum of treelevel-like objects integrated over a proper phase-space region. From the physical point of view, loop particles are converted into real-radiation ones. From the mathematical side, the integration domain is transformed from a Minkowski to an Euclidean space. In fact, the numerical evaluation of multiloop integrals through LTD is, with respect to the approaches that pass by Feynman parametrization or Mellin-Barnes transformations, more efficient as the number of integrations to be performed does not scale with the number of external particles. Very recently, a novel LTD-inspired representation of multiloop multileg scattering amplitudes was presented in Refs. [14-17,19,20]. This strategy, based on the nested residue strategy, leads to very compact integrand-level representations, which are free of unphysical or noncausal singularities. Likewise, alternative studies of LTD have been presented in Refs. [21-26].

In this paper, we apply the LTD formalism to the calculation of multiloop helicity amplitudes. We exploit the fact that LTD works at the level of denominators, and the structure of the numerator does not generate any additional difficulties. To this end, we start considering 
illustrative examples in which the simplicity of the latter is displayed. We make use of the spinor-helicity formalism, where we write definite helicity states for the external particles. On top of it, we also use the momentum twistors' variables [27] to implement simplifications in the integrand of the helicity amplitudes. These variables, due to their mathematical properties, allow us to express any kinematic process in terms of the minimal set of variables. In other words, for a process with $N$ external massless particles we have $3 N-10$ invariants to deal with $[28,29]$. Likewise, the extension to massive particles is straightforward.

Besides the clearness LTD offers us to compute any multiloop amplitude, in this paper, we also insist on the local UV renormalization. Hence, for the sake of simplicity, we consider scattering amplitudes that are IR and UV safe but might still exhibit a local singular UV behavior. For the latter, it is known that UV finite integrals might be locally divergent in the high-energy region [30]. Therefore, a careful treatment in the UV has to be performed. For instance, at one-loop level, we refer the reader to Refs. [31-35] (and references therein) and to Ref. [36] beyond one loop. We remark that the idea of performing a local UV renormalization is to obtain welldefined integrands in four space-time dimensions that allow a straightforward numerical evaluation.

The paper is organized as follows. In Sec. II, we recall the basis of the LTD formalism, with special emphasis on the formulae applied in this work. We briefly present its extension to the multiloop case. In Sec. III, we provide a description of the generation of kinematical variables by using the spinor-helicity formalism. In Sec. III A, we focus on the parametrization of the loop three-momenta to integrate the dual contributions when external momenta are complex. The introduction of local UV renormalization counterterms is reviewed in Sec. IV. The main part of this manuscript is presented in Sec. V, where we show numerical results for explicit examples at one-loop level. Special emphasis is put on the local cancellation of UV singularities, to render the expressions integrable in four space-time dimensions. Then, we present a careful study of the local UV counterterms for $H \rightarrow g g$ at two loops in Sec. VI. For this purpose, we make use of the computational tools developed throughout this paper. This allows us to support the feasibility of the LTD-based numerical strategy with realistic scattering processes, as well as its efficiency. Conclusions and future research directions are analyzed in Sec. VII.

\section{LOOP-TREE DUALITY IN A NUTSHELL}

The LTD theorem [9-17] rewrites any loop integral in terms of tree-level-like expressions that correspond to cutting, i.e., setting on shell, a number of internal particles equal to the number of loops. It relies on a suitable application of the Cauchy's residue theorem to reduce one degree of freedom for each loop. We usually apply it on the energy component of the loop momenta, which translates into reducing the original Minkowski integration domain into the Euclidean space of the loop threemomenta, although it could be used to remove any other component of the loop momenta.

In order to explain the formalism, let us consider a generic $L$-loop $N$-particle scattering amplitude, where the external momenta are labeled as $p_{i}$ with $i \in\{1, \ldots, N\}$. We have $L$ independent primitive integration variables, $\left\{\ell_{j}\right\}_{j=1, \ldots, L}$, and the momenta associated to the different internal lines can be written as $q_{i_{s}}=\ell_{s}+k_{i_{s}}$, where $\ell_{s}$ is a linear combination of the primitive loop momenta, and $k_{i_{s}}$ is a linear combination of external momenta. All the propagators depending on the same linear combination of primitive loop momenta $\ell_{s}$ are enclosed together inside the set $s$. With this notation, a generic amplitude is given by

$$
\mathcal{A}_{N}^{(L)}(1, \ldots, n)=\int_{\ell_{1}, \ldots, \ell_{L}} \sum \mathcal{N} \times G_{F}(1, \ldots, n),
$$

where $\mathcal{N}$ represents an arbitrary numerator, and

$$
G_{F}(1, \ldots, n)=\prod_{j \in 1 \cup \ldots \cup n}\left(G_{F}\left(q_{j}\right)\right)^{\alpha_{j}}
$$

is a product of Feynman propagators spanned over all the possible momenta sets. Each scalar Feynman propagator can be written as

$$
G_{F}\left(q_{i}\right)=\frac{1}{q_{i}^{2}-m_{i}^{2}+\imath 0}=\frac{1}{q_{i, 0}^{2}-\left(q_{i, 0}^{(+)}\right)^{2}},
$$

where $q_{i, 0}^{(+)}=\sqrt{\boldsymbol{q}_{i}^{2}+m_{i}^{2}-\imath 0}$ corresponds to the positive on-shell energy of the associated internal particle. In Eq. (2), we also take into account the possibility of arbitrary powers of the propagators, through the parameters $\left\{\alpha_{j}\right\}$. Regarding the integration measure, we have

$$
\int_{\ell} \equiv-\imath \mu^{4-d} \int \frac{d^{d} \ell}{(2 \pi)^{d}},
$$

with $\mu$ an arbitrary energy scale to restore the proper units after the extension to a $d$-dimensional space-time.

The LTD representation is obtained by defining the nested residues and closing the integration contour in the lower part of the complex plane. Explicitly, if $\mathcal{A}_{F}^{(L)}$ represents the integrand of Eq. (1) in the Feynman representation, then the first iteration of Cauchy's theorem leads to

$$
\mathcal{A}_{D}^{(L)}(1 ; 2, \ldots, n)=\sum_{i_{1} \in 1} \operatorname{Res}\left(\mathcal{A}_{F}^{(L)}, \operatorname{Im}\left(\eta \cdot q_{i_{1}}\right)<0\right),
$$

where we sum over all the possible configurations containing one on-shell propagator of the first set 1 , whilst propagators in the remaining sets are left off shell. In this expression, $\eta$ is 
a futurelike vector that determines which degree of freedom of the loop momenta is integrated out. For the sake of simplicity, we choose $\eta^{\mu}=(1, \overrightarrow{0})$, which corresponds to apply the nested residues on the energy component of the propagator's momenta $q_{i, 0}$. After the $r$ th iteration, we end up with

$$
\begin{aligned}
& \mathcal{A}_{D}^{(L)}(1, \ldots, r ; r+1, \ldots, n) \\
& \quad=\sum_{i_{r} \in r} \operatorname{Res}\left(\mathcal{A}_{D}^{(L)}(1, \ldots, r-1 ; r, \ldots, n), \operatorname{Im}\left(\eta \cdot q_{i_{r}}\right)<0\right),
\end{aligned}
$$

where all the propagators in the sets to the right of the semicolon on the lhs of Eq. (6) are left off shell, and we set exactly one propagator on shell in each one of the first $r$ sets. Once we apply the Cauchy's theorem in the energy component of the primitive momenta, the integration measure turns into

$$
\int_{\ell} \rightarrow \int_{\vec{\ell}} \equiv-\mu^{d-4} \int \frac{d^{d-1} \ell}{(2 \pi)^{d-1}}
$$

i.e., transforming the $d$-dimensional Minkowski space into a $(d-1)$-dimensional Euclidean one. The dual LTD representation is obtained after the $L$ th iteration of the residue computation, i.e.,

$$
\mathcal{A}_{N}^{(L)}(1, \ldots, n)=\int_{\vec{e}_{1} \ldots \vec{e}_{L}} \sum_{\sigma} \mathcal{A}_{D}^{(L)}\left(\sigma_{1}, \ldots, \sigma_{L} ; \sigma_{L+1}, \ldots, \sigma_{n}\right),
$$

where we sum over all the possible combinations of simultaneous $L$ cuts in different momenta sets. This is equivalent to perform as many cuts as loops, in order to open the loop amplitude into a set of nondisjoint trees [9].

At one loop, the aforementioned formulae reduce to the usual LTD representation given in Refs. $[9,10]$. It can be easily obtained by summing over all the possible single cuts and replacing the Feynman propagators by the so-called dual propagators, namely

$$
G_{D}\left(q_{i} ; q_{j}\right)=\frac{1}{q_{j}^{2}-m_{j}^{2}-\imath 0 \eta \cdot k_{j i}},
$$

where $q_{j}$ is the momenta flowing through the line, $q_{i}$ corresponds to the one that is set on shell, and $k_{j i}=q_{j}-q_{i}$. As in Eq. (5), $\eta$ is a generic futurelike vector, which is usually chosen as $\eta^{\mu}=(1, \overrightarrow{0})$. It is important to notice that the dual prescription accounts for the information contained in the multiple cuts defined within the Feynman tree theorem [37]. Also, that within the representation introduced in Refs. [14-17], there is no need to explicitly deal with the complex prescription of the dual

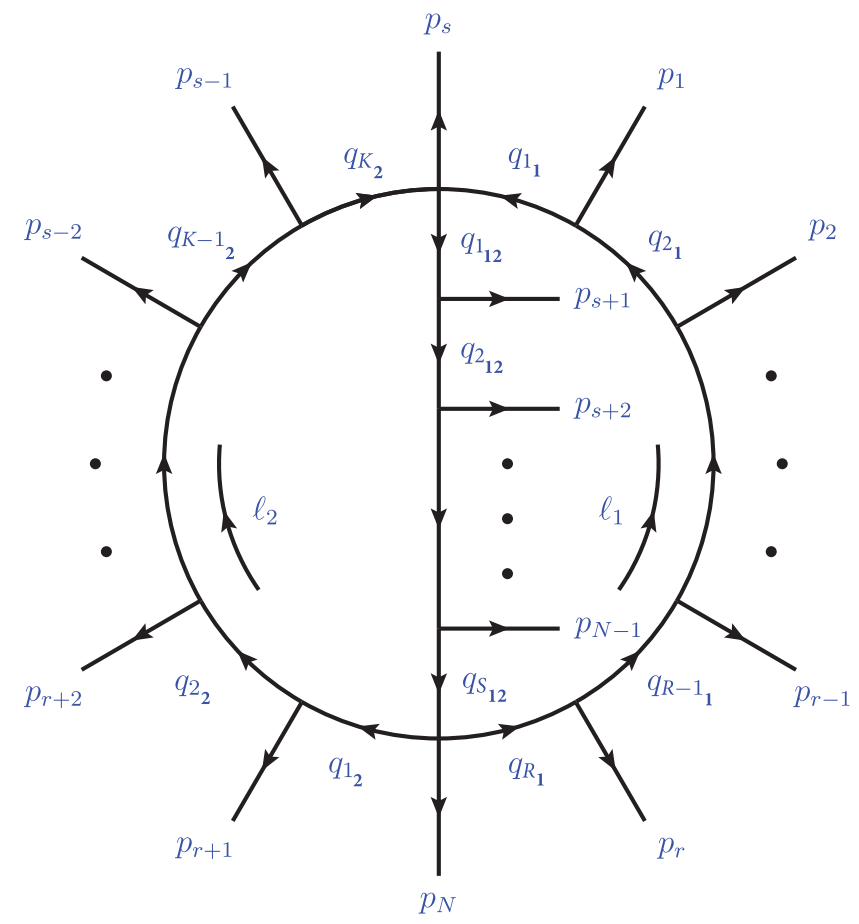

FIG. 1. Diagrammatic representation of a generic two-loop diagram with $N$ external particles.

propagators since this operation is encoded within the definition of the nested residues.

In this paper, we present practical applications up to the two-loop level, although the underlying algorithms can be extended to any loop order. In the particular two-loop case, we have $\ell_{j}=\left\{\ell_{1}, \ell_{2}\right\}$, and there are three sets of propagators, $s \in\{1,2,12\}$, with $q_{i_{12}}=\ell_{1}+\ell_{2}+k_{i_{12}}$, as shown in Fig. 1. More details about the underlying subtleties of the two-loop case are available in Refs. $[10,36]$.

\section{A. Multiple poles and IBPs}

Multiloop integrals and local UV counterterms can contribute with multiple powers of the propagators. Using integration-by-parts identities (IBPs) [38-40], they can be reduced to linear combinations of other integrals containing only single powers of the propagators, as done in [11]. However, this modifies the local behavior of the integrands and might spoil the point-by-point cancellation of IR singularities present in the real-emission contribution. Thus, we will stick to the local approach and avoid using IBPs.

The master formula given in Eq. (8) handles also amplitudes with multiple powers of the propagators since its definition relies directly on the nested application of the Cauchy's theorem. A careful discussion about the computation of the residue is presented in Refs. $[9,11]$. It is important to take care of the dual prescription for the contributions associated to the original amplitude since it may contain thresholds in the low energy region. In that 
case, the propagators associated to off-shell propagators must be promoted to dual propagators. In contrast, when applying the LTD formalism to the UV counterterms, we can neglect the complex prescriptions and straightforwardly use the Cauchy's formula for computing the residue.

It is worth noticing that compact formulae to obtain the LTD representation with higher powers are presented in Ref. [15]. These expressions are obtained considering the derivatives, with respect to $\left(q_{i, 0}^{(+)}\right)^{2}$, and taking advantage of Eq. (3) to express propagators in terms of on-shell energies.

\section{GENERATION OF THE KINEMATICS}

In order to provide helicity amplitudes, we take advantage of the momentum twistor parametrization proposed in Ref. [27], where the standard spinor products, $\langle\bullet \bullet\rangle,[\bullet \bullet$, are replaced by a minimal set of independent variables $z_{i}$. The number of variables in the latter depends on the kinematic process. In particular, any $n$-point massless amplitude can be expressed in terms of $3 n-10$ independent variables. Hence, the extension to amplitudes with massive particles is straightforward. In Appendix A, we briefly recall the main features of these variables.

Within the LTD approach, the evaluation of integrals is performed in the momentum space instead of using Feynman parameters or, equivalently, Mellin transformations. Then, the most suitable way of computing helicity amplitudes is through the form factors' decomposition, which has been applied within the LTD framework in Refs. [30,36]. Very recently, some alternative methods to bypass this decomposition were proposed $[41,42]$. Since the representation of the polarization vectors may be an obstacle depending on the regularization scheme being applied, we use the one in which external wave functions are kept in four dimensions, i.e., 't Hooft-Veltman (HV) [43] and four-dimensional helicity (FDH) $[44,45]$.

The use of $\mathrm{HV}$ and FDH allows us to project objects in $d$ dimensions into a four-dimensional space by making use of the following properties [49]:

$$
\begin{aligned}
& q_{i,[d]} \cdot p_{j,[4]}=q_{i,[4]} \cdot p_{j,[4]}, \\
& q_{i,[d]} \cdot \varepsilon_{j,[4]}=q_{i,[4]} \cdot \varepsilon_{j,[4]},
\end{aligned}
$$

where we contracted the loop momentum with external momenta or polarization vectors. Therefore, we only need to keep track of squared loop momenta, $q_{i,[d]} \cdot q_{j,[d]}$. It turns out that due to the cuts performed within the LTD formalism, we can easily remove this dependence and work with objects in four dimensions. Let us also remark that, within this approach, we do not need to include extradimensional products, i.e., $q_{i,[d-4]} \cdot q_{j,[d-4]}$.

Then, working in four space-time dimensions, we can parametrize the loop momenta in terms of a fourdimensional basis, i.e., $\mathcal{E}=\left\{e_{i}\right\}$. Therefore, to reduce as much as possible the number of scalar products to be evaluated, we choose $\mathcal{E}=\left\{p_{1}, p_{2}, \varepsilon_{12}, \varepsilon_{21}\right\}$. With this choice, the loop momenta is expressed as

$$
q_{i}^{\alpha}=x_{i, 1} p_{1}^{\alpha}+x_{i, 2} p_{2}^{\alpha}+x_{i, 3} \varepsilon_{12}^{\alpha}+x_{i, 4} \varepsilon_{21}^{\alpha},
$$

where $p_{1}$ and $p_{2}$ are the massless momenta built from the parametrization obtained from the momentum twistors of an $n$-point kinematics and $\varepsilon_{i j}^{\alpha}=\frac{1}{2}\left\langle i\left|\gamma^{\alpha}\right| j\right]$. We remark that for the elements of the basis, we explicitly work with the components of the four-vectors. Hence, with this decomposition, all the scalar products involving external momenta or polarization vectors contracted with the loop momenta can always be expressed in terms of scalar products among the elements of the basis and the loop momenta, i.e., $q_{i} \cdot e_{j}$. The aim of this refinement is twofold, firstly, to reduce the number of scalar products required for the computation, and in the second place, to cancel redundant expressions that appear at integrand level. This prevents some noncontributing terms that pop up in intermediate steps of the computation before performing an explicit evaluation.

\section{A. Parametrization of the loop momentum}

As discussed in Sec. II, once LTD is applied to any loop integral or virtual amplitude, the integration over the loop energy component is removed, and the remaining one is performed over an Euclidean space. Thus, the loop threemomentum needs to be properly parametrized to improve the computational efficiency. We remark that we are considering a complex-valued parametrization of the external momenta. Explicitly, the second component of the three-momentum is purely imaginary; this is due to the method applied to build their representation starting from scalar invariants [50]. It is worth noticing, however, that the scalar products among themselves do not contain any complex phase (i.e., they are purely real), as expected in any physical kinematic configuration. Hence, to overcome any possible issue when using a real parametrization of the loop three-momentum, we express it in cylindrical coordinates,

$$
\boldsymbol{\ell}_{i}=\left(\xi_{i} \cos \phi_{i}, \rho_{i}, \xi_{i} \sin \phi_{i}\right),
$$

for the ith loop three-momentum. Then, the resulting integral is given by

$$
I_{i}=\int_{0}^{\infty} \xi_{i} d \xi_{i} \int_{0}^{2 \pi} d \phi_{i} \int_{-\infty}^{\infty} d \rho_{i} \mathcal{I}_{i}\left(\xi_{i}, \phi_{i}, \rho_{i}\right),
$$

where $\mathcal{I}_{i}$ is the integrand after plugging the explicit parametrization of the loop three-momentum (12). We note that carefully integrating $\mathcal{I}_{i}$ over $\rho_{i}$ brings large cancellations, in particular, when considering kinematical configurations below threshold. This is because the imaginary part introduced by the prescriptions must cancel in 
these configurations, and the $\rho_{i}$ variable captures all the imaginary contributions due to the explicit functional form of the parametrization of the external momenta. Of course, we are excluding from this claim the presence of imaginary terms introduced by the numerators (for instance, originated by the polarization vectors). Hence, to account for the simplifications that occur in the $\rho_{i}$ integration, we rewrite Eq. (13) as

$$
\begin{aligned}
I_{i}= & \int_{0}^{\infty} \xi_{i} d \xi_{i} \int_{0}^{2 \pi} d \phi_{i} \int_{0}^{\infty} d \rho_{i} \\
& \times\left[\mathcal{I}_{i}\left(\xi_{i}, \phi_{i}, \rho_{i}\right)+\mathcal{I}_{i}\left(\xi_{i}, \phi_{i},-\rho_{i}\right)\right],
\end{aligned}
$$

which turns out to be equivalent to consider the real-part of the integrand in the previously mentioned conditions.

Furthermore, we notice that the $\left(\xi_{i}, \rho_{i}\right)$ - plane can be compactified by changing variables and using polar coordinates. Explicitly, we define

$$
\left(\xi_{i}, \rho_{i}\right) \rightarrow \frac{x_{i}}{1-x_{i}}\left(\cos \theta_{i}, \sin \theta_{i}\right)
$$

with $0 \leq x_{i}<1$ and $0 \leq \theta_{i}<\pi / 2$. In the last part, we restricted the angular integration to the first quadrant because both $\xi_{i}$ and $\rho_{i}$ are positive.

\section{LOCAL UV RENORMALIZATION}

Since we are aiming for a complete numerical implementation, it is necessary to build integrand-level counterterms, in order to cancel the local singular behavior at very high energies of the amplitudes under consideration. In the following, we recall how to generate these counterterms very easily from the original amplitudes $[30,36]$.

For a given loop momentum $\ell_{j}$, we consider the integrand-level replacement

$$
\mathcal{S}_{j, \mathrm{UV}}:\left\{\ell_{j}^{2} \mid \ell_{j} \cdot k_{i}\right\} \rightarrow\left\{\lambda^{2} q_{j, \mathrm{UV}}^{2}+\left(1-\lambda^{2}\right) \mu_{\mathrm{UV}}^{2} \mid \lambda q_{j, \mathrm{UV}} \cdot k_{i}\right\},
$$

where $\mu_{\mathrm{UV}}$ is an arbitrary scale that can be identified with the renormalization scale and $q_{j, \mathrm{UV}}=\ell_{j}+k_{j, \mathrm{UV}}$. The vector $k_{j, \mathrm{UV}}$ is arbitrary, and we can set $k_{j, \mathrm{UV}} \equiv 0$ without any loss of generality.

By applying $\mathcal{S}_{j, \mathrm{UV}}$ to an unintegrated and uncut one-loop amplitude $\mathcal{A}_{N}^{(1)}$ with loop momentum $\ell_{j}$, and then expanding in $\lambda$ around infinity up to logarithmic degree (this operation will be represented by the operator $L_{\lambda}$ in the following), we directly obtain an integrand-level expression that cancels the local UV singularities exhibited by $\mathcal{A}_{N}^{(1)}$. This procedure is equivalent to expanding around the UV propagator $[31,32,34,35]$, i.e.,

$$
G_{F}\left(q_{j, \mathrm{UV}}\right)=\frac{1}{q_{j, \mathrm{UV}}^{2}-\mu_{\mathrm{UV}}^{2}+i 0},
$$

and then keeping only the most divergent terms: the resulting object mimics locally the UV behavior of the original expression. It is important to note, though, that this counterterm may generate a finite part after integration, which must be fixed through a scheme fixing parameter $d_{j, \mathrm{UV}}$. Therefore, the counterterm reads,

$\mathcal{A}_{j, \mathrm{UV}}^{(1)}=L_{\lambda}\left(\left.\mathcal{A}_{N}^{(1)}\right|_{\mathcal{S}_{j, \mathrm{UV}}}\right)-d_{j, \mathrm{UV}} \mu_{\mathrm{UV}}^{2} \int_{\ell_{j}}\left(G_{F}\left(q_{j, \mathrm{UV}}\right)\right)^{3}$,

where the integral multiplying $d_{j, \mathrm{UV}}$ integrates to the same finite quantity in both four and $d$ space-time dimensions. By construction, the first term in the rhs of Eq. (18) exactly contains the UV divergent part of the original amplitude, extracted through the operator $L_{\lambda}$ at integrand level. Thus, the quantity $\mathcal{A}_{j, \mathrm{UV}}^{(1)}$ locally cancels the UV behavior of $\mathcal{A}^{(1)}$, while giving the required finite part (which is 0 , for instance, in the $\overline{\mathrm{MS}}$ scheme).

For a two-loop amplitude $\mathcal{A}_{N}^{(2)}$, the general local renormalization procedure has been extended in Ref. [36]. In the two-loop case, it is necessary to consider three UV divergent configurations involving the two internal momenta, $\ell_{1}$ and $\ell_{2}$. For instance, we can consider the regimes

$\left\{\begin{array}{l}\left|\boldsymbol{\ell}_{1}\right| \rightarrow \infty \\ \left|\boldsymbol{\ell}_{2}\right| \text { fixed }\end{array}, \quad\left\{\begin{array}{l}\left|\boldsymbol{\ell}_{1}\right| \text { fixed } \\ \left|\boldsymbol{\ell}_{2}\right| \rightarrow \infty\end{array}, \quad\left\{\begin{array}{l}\left|\boldsymbol{\ell}_{1}\right| \rightarrow \infty \\ \left|\boldsymbol{\ell}_{2}\right| \rightarrow \infty\end{array}\right.\right.\right.$.

The counterterms relative to the singular behavior of the first two regimes can be generated using the replacement in Eq. (16). These contributions are known as the single UV counterterms because only one integration loop momenta goes to infinity, whilst the other remains subdominant. To obtain the local counterterm needed to cancel the third one, we need the additional replacement,

$$
\begin{aligned}
\mathcal{S}_{\mathrm{UV}^{2}} & : \\
\ell_{j}^{2} & \rightarrow \lambda^{2} q_{j, \mathrm{UV}}^{2}+\left(1-\lambda^{2}\right) \mu_{\mathrm{UV}}^{2}, \\
\ell_{j} \cdot \ell_{k} & \rightarrow \lambda^{2} q_{j, \mathrm{UV}} \cdot q_{k, \mathrm{UV}}+\left(1-\lambda^{2}\right) \mu_{\mathrm{UV}}^{2} / 2, \\
\ell_{j} \cdot k_{i} & \rightarrow \lambda q_{j, \mathrm{UV}} \cdot k_{i},
\end{aligned}
$$

to build the counterterm

$$
\begin{aligned}
\mathcal{A}_{\mathrm{UV}^{2}}^{(2)}= & L_{\lambda}\left(\left.\left(\mathcal{A}_{N}^{(2)}-\sum_{j=1,2} \mathcal{A}_{j, \mathrm{UV}}^{(2)}\right)\right|_{S_{\mathrm{UV}^{2}}}\right) \\
& -d_{\mathrm{UV}^{2}} \mu_{\mathrm{UV}}^{4} \int_{\ell_{1} \ell_{2}}\left(G_{F}\left(q_{1, \mathrm{UV}}\right)\right)^{3}\left(G_{F}\left(q_{2, \mathrm{UV}}\right)\right)^{3}
\end{aligned}
$$

where once again, the term proportional to the schemefixing coefficient $d_{\mathrm{UV}^{2}}$ integrates to a finite quantity. 
This last terms accounts for the so-called double UV divergence, i.e., when both integration loop momenta go to infinity.

The one- and two-loop versions of this algorithm were explicitly implemented in a MATHEMATICA code [36]. It is fully process independent and can be directly applied to any scattering amplitude, producing the appropriate local counterterm to regularize the divergent behavior in the high-energy region.

\section{APPLICATIONS AT ONE LOOP}

In this section, we give explicit examples in which the techniques described in Secs. III and IV are applied. We explore the computational advantages of the LTD-based representation combined with the spinor-helicity formalism, which constitutes the central part of this paper. We focus on processes that contain two to four kinematic invariants, and we consider the nonvanishing helicity configurations. We summarize the description of our examples in Table I. In the following, the kinematic invariants are implicitly given in $\mathrm{GeV}^{2}$.

Since we are aiming at a calculation performed purely in four space-time dimensions, we restrict the analysis presented in this paper to helicity amplitudes that are simultaneously IR and UV finite. Although the processes under consideration exhibit these features, they might still posses a local UV-divergent behavior that prevents to perform the calculation directly in four space-time dimensions, without introducing any additional regularization. This is because, in the most general case, the associated integrands turn out to be nonintegrable functions in the high-energy limit (or UV limit).

Eventually, in the context of dimensional regularization, setting $d=4$ from the beginning of the calculation can generate wrong results. This situation was exhaustively discussed in Ref. [30] for the computation of the decay width of $H \rightarrow \gamma \gamma$ at leading order. Therefore, we need to build local UV counterterms that take care of the singularities that appear at integrand level in the UV limit. In other words, we need to locally renormalize our amplitude, as explained in Sec. IV, to render the expressions integrable in four space-time dimensions.

The calculation of the amplitude $H \rightarrow \gamma \gamma$ performed in Ref. [30], through the form factor decomposition, exploited

TABLE I. Processes considered at one-loop level with their kinematic scales. We indicate the nonvanishing helicity configurations.

\begin{tabular}{lcc}
\hline \hline Process & Kinematic scales & Helicity configuration \\
\hline$H \rightarrow \gamma \gamma$ & $s_{12}, m_{f}^{2}$ & ++ \\
$\gamma \gamma \rightarrow \gamma \gamma$ & $s, t, m_{f}^{2}$ & ++++-+++--++ \\
$H \rightarrow g g g$ & $s_{12}, s_{13}, s_{23}, m_{f}^{2}$ & +++-++ \\
\hline \hline
\end{tabular}

several analytical properties in order to simplify the results. In particular, due to gauge invariance, it was possible to remove vanishing terms at integrand level. In contrast, in the present calculation, we directly generate the proper UV counterterm to render the amplitude integrable in four space-time dimensions, without taking into account any kind of analytical property to achieve further simplifications. The numerical integration performed by LTD was compared with the analytic expression of the amplitude. For the latter, we rely on two MATHEMATICA packages, the integral reduction provided by FEYNCALC [51-53] and the analytic expressions for the one-loop scalar integrals collected in PACKAGE-X [54]. Our results are shown in Fig. 2, where we plot the value of the amplitude as a function of the fermion internal mass $m_{f}^{2}$ for different values of $s_{12}$. An excellent agreement is found, as expected from our previous studies of this process $[30,36]$. Regarding the scale of the vertical axis of Fig. 2, we used the default normalization provided by the aforementioned packages, and all the invariants are expressed in $\mathrm{GeV}^{2}$. The same choice is applied for the remaining plots shown in this article.

For the processes including more kinematic scales, namely $\gamma \gamma \rightarrow \gamma \gamma$ and $H \rightarrow g g g$, we do not rely on FEYNCALC because it becomes inefficient when the rank of the loop momentum in the numerator starts increasing. Therefore, instead of decomposing the integrals, we work at the integrand level by reducing the amplitudes to scalar one-loop integrals. In order to do so, we follow the Ossola-Papadopoulos-Pittau method [55] together with the integrand reduction algorithm [56-63]. For the evaluation of the scalar one-loop integrals, we keep using PACKAGE-X. Our results are shown in Figs. 3 and 4, where we plot the amplitudes as a function of the fermion

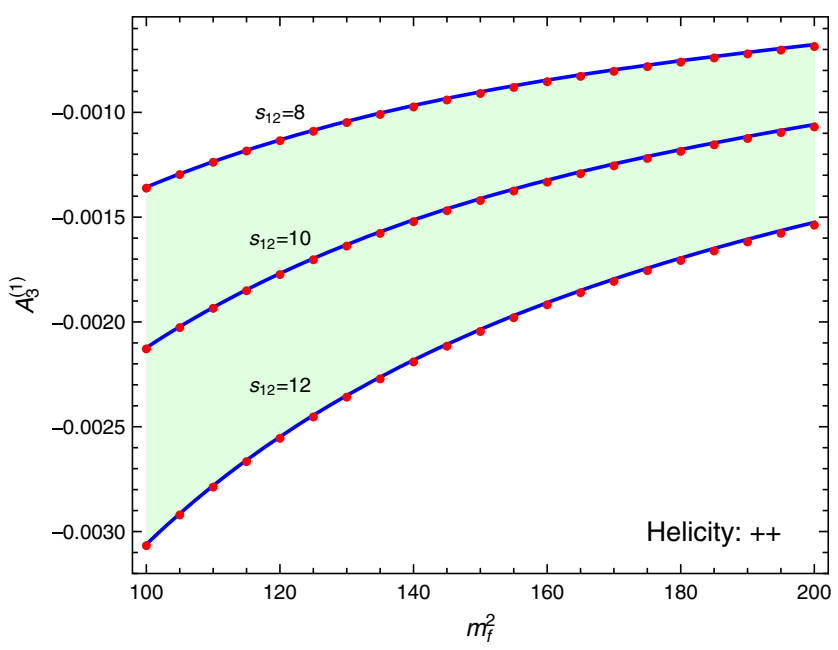

FIG. 2. $\quad H \rightarrow \gamma \gamma$ at one loop as a function of the internal mass $m_{f}^{2}$. We plot the predictions for $s_{12} \in\{8,10,12\}$. The solid blue lines correspond to the analytical results, while the red points are computed through the LTD-based numerical approach. 

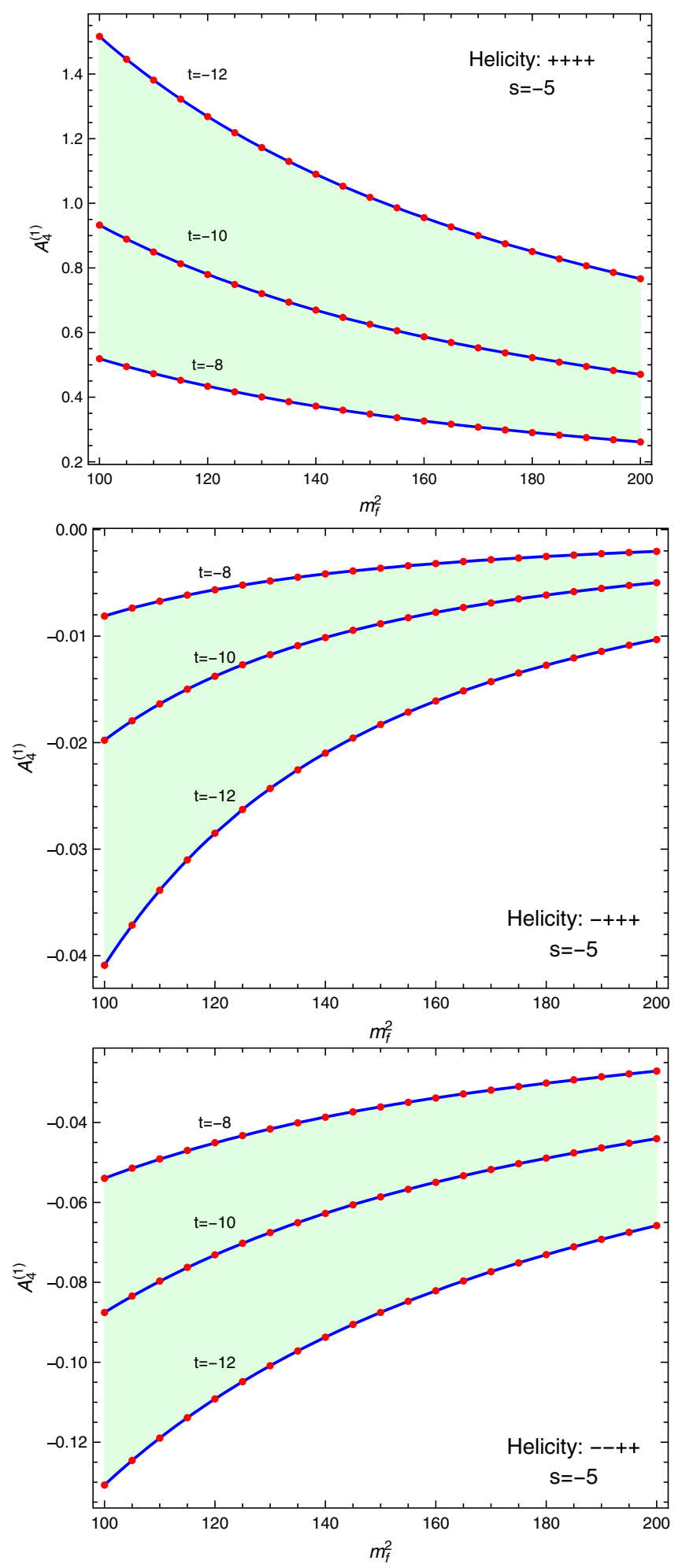

FIG. 3. One-loop contributions to the process $\gamma \gamma \rightarrow \gamma \gamma$, as a function of the internal mass $m_{f}^{2}$. We consider all the possible helicity configurations for a fixed ordering of the external legs: ,++++-+++ and --++ . In each case, we fix $s=-5$ and plot the predictions for $t \in\{-8,-10,-12\}$. The solid blue lines correspond to the analytical results, while the red points were computed through the LTD-based numerical approach. internal mass $m_{f}^{2}$. For $\gamma \gamma \rightarrow \gamma \gamma$, we fixed $s=-5$ and considered $t=\{-8,-10,-12\}$. In the case of $H \rightarrow g g g$, $s_{12}=-1 / 3$ and $s_{23}=-1 / 7$ remained fixed, while we varied $s_{13} \in[8,12]$. The agreement is very good for both processes, in all the kinematical and helicity configurations that we explored. Small numerical instabilities arise for $m_{f}^{2}>180$ in $H \rightarrow g g g g$, although they can be fixed by slightly increasing the numerical precision of the integration.

Let us stress that in the processes we consider within the LTD approach, we do not perform any integral or integrand reduction. We directly evaluate them with the proper inclusion of the UV local counterterms, as explained in Sec. IV. Regarding the evaluation of the required integrals,
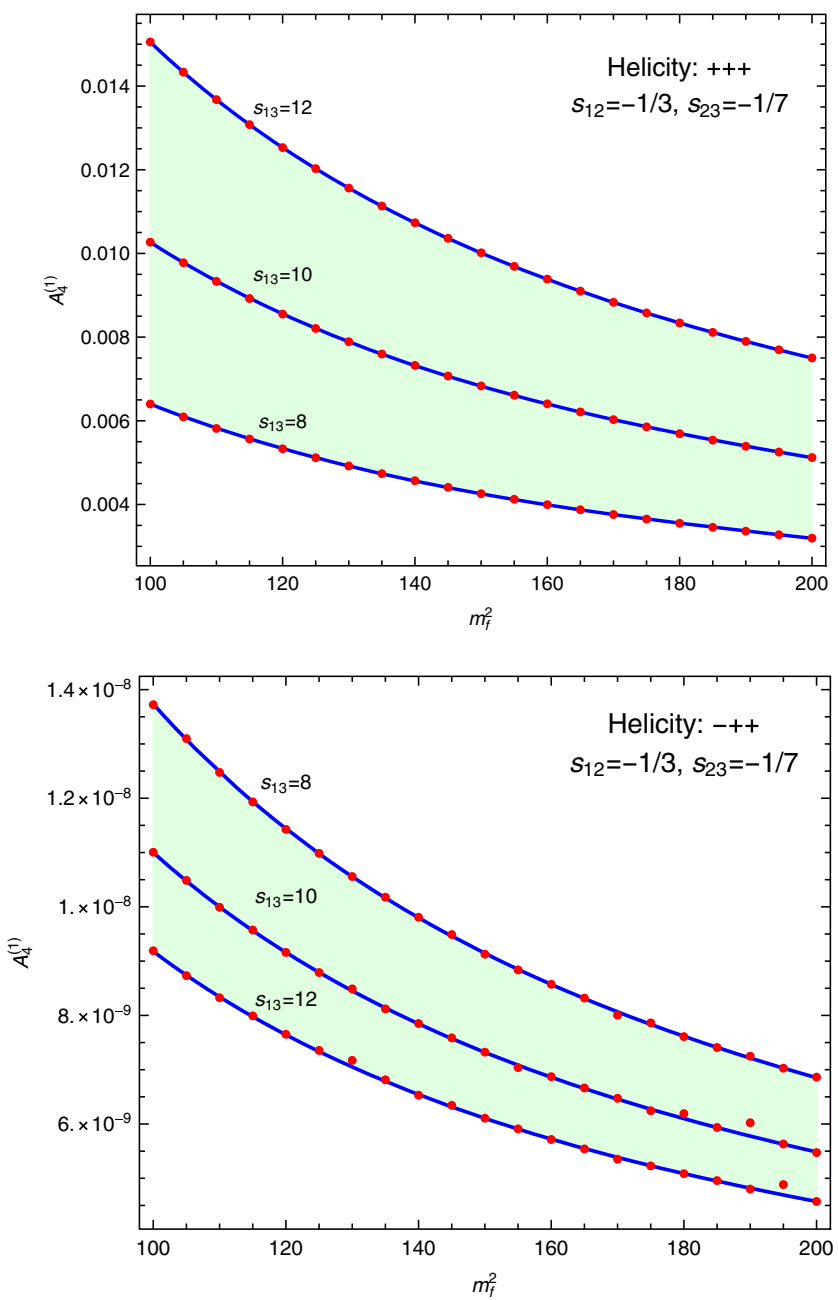

FIG. 4. One-loop contributions to the process $H \rightarrow g g g$, as a function of the internal mass $m_{f}^{2}$. We consider all the possible configurations for a given helicity amplitude, +++ and -++ . In each case, we show the predictions for $s_{13}=\{8,10,12\}$. The solid blue lines corresponds to the analytical results, while the red points were computed through the LTD-based numerical approach. 
we use the built-in MATHEMATICA function NIntegrate on a desktop machine with an Intel i7 $(3.4 \mathrm{GHz})$ processor with eight cores and $16 \mathrm{~GB}$ of RAM. The computing time for each phase-space point was $\mathcal{O}\left(30^{\prime \prime}\right)$. It is worth appreciating that we did not implement any further optimization for performing the numerical integration. In future developments, we plan to improve this point, by using parallel integration strategies or optimized integrators to speed up the computation. In any case, we would like to highlight that the main advantage of our framework relies on the smooth behavior of the integrand and the fully local cancellation of singularities.

\section{A TWO-LOOP EXAMPLE: $H \rightarrow g g$}

In the previous section, we have demonstrated the viability of the LTD approach to tackle one-loop locally UV-divergent helicity amplitudes in four space-time dimensions. Here, we will show that it is also a reliable strategy for two-loop processes. So, we focus on the computation of $\mathcal{O}\left(e^{3} g_{S}^{2}\right)$ corrections to the decay process $H \rightarrow g g$, where the internal particles are massive top quarks and $Z$ bosons. In this case we are dealing with a finite amplitude, whose contributions are given by the diagrams shown in Fig. 5 . There are neither IR nor UV singularities, but it is mandatory to perform a local renormalization to smoothly pass from $d$ to four space-time dimensions when evaluating this amplitude.

In the same spirit of Ref. [36], we start considering a minimal set of independent denominators. This is done in order to match the structures of the planar $(\mathrm{P})$ and nonplanar (NP) contributions at integrand level. Hence, we express all Feynman diagrams in terms of the following families of integrals,

$$
I_{\mathrm{P} / \mathrm{NP}}=\int_{\ell_{1}} \int_{\ell_{2}} \frac{1}{D_{1}^{\nu_{1}} D_{2}^{\nu_{2}} D_{3}^{\nu_{3}} D_{4}^{\nu_{4}} D_{5}^{\nu_{5}} D_{6}^{\nu_{6}} D_{7}^{\nu_{7}}},
$$

with

$$
\begin{aligned}
& D_{1}=\left(\ell_{1}+\ell_{2}\right)^{2}-m_{Z}^{2}+\imath 0, \\
& D_{2}=\left(\ell_{1}+\ell_{2}+p_{1}+p_{2}\right)^{2}-m_{Z}^{2}+\imath 0 \\
& D_{3}=\ell_{1}^{2}-m_{t}^{2}+\imath 0 \\
& D_{4}=\left(\ell_{1}+p_{1}\right)^{2}-m_{t}^{2}+\imath 0 \\
& D_{5}=\left(\ell_{1}+p_{1}+p_{2}\right)^{2}-m_{t}^{2}+\imath 0 \\
& D_{6}=\ell_{2}^{2}-m_{t}^{2}+\imath 0 \\
& D_{7}=\left(\ell_{2}-p_{1}\right)^{2}-m_{t}^{2}+\imath 0
\end{aligned}
$$

where the auxiliary propagators are $D_{7}$ and $D_{3}$ in the planar and nonplanar topologies, respectively. In the previous expressions, we also consider the presence of nontrivial numerators by allowing $\nu_{i}<0$.

In the following, we discuss the extraction and the structure of the single and double UV local counterterms. In particular, we have

(i) Single UV counter-terms,

$$
\mathcal{A}_{1, \mathrm{UV}}^{(2)}=\mathcal{A}_{2, \mathrm{UV}}^{(2)}=0 .
$$

Let us remark that single UV counterterms vanish at integrand level, as a consequence of the way in which the propagators have been labeled. A different choice might lead to nonvanishing integrand-level expressions. In order to profit from this property, we labeled the propagators in such a way that the most UV-divergent contributions depend on $\ell_{1}$ and $\ell_{2}$, whilst those contributions depending on $\ell_{1}+\ell_{2} \equiv \ell_{12}$ exhibit a less UV-divergent behavior.

(ii) Double UV counterterm,
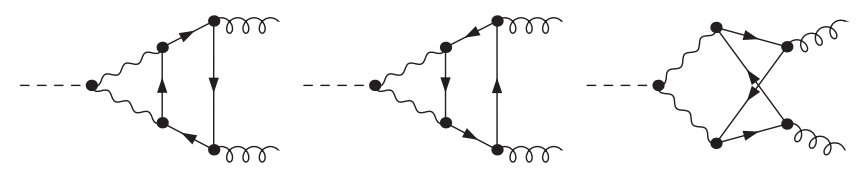

FIG. 5. Two-loop diagrams for the process $H \rightarrow g g$ with internal massive top quarks and $Z$ bosons. where $\delta^{a_{1} a_{2}}$ accounts for the color factor originated from the two external gluons, and $g_{f}=$ $e g g^{\prime} g_{S}^{2} m_{Z}\left(g_{L}^{2}+g_{R}^{2}\right)$ corresponds to the overall coupling constant. In order to refer to the UV limit of the Feynman propagators, we introduced the shorthand notation 


$$
D_{j \mathrm{UV}}=\left(G_{F}\left(q_{j, \mathrm{UV}}\right)\right)^{-1},
$$

with $q_{j, \mathrm{UV}}=\ell_{j}$ with $j=\{1,2\}$, and $q_{12, \mathrm{UV}}=\ell_{12}$.

It is easy to check that after applying integrationby-parts identities on $\mathcal{A}_{\mathrm{UV}^{2}}^{(2)}$, it vanishes in $d$ dimensions. In fact, as expected from the finiteness of the amplitude, the UV singularities that emerge from the planar and nonplanar diagrams cancel exactly at this point. The latter is indeed in agreement with the above discussion since we are including an additional term that does not alter the behavior in $d$ dimensions, but it collects several local features in $d=4$. In other words, we end up with an integrable function in four space-time dimensions.

Subtracting the counterterms in Eqs. (24) and (25) from the original $H \rightarrow g g$ amplitude, we tested the UV limit [64]. We parametrized the loop momenta and studied the large energy limit by performing a series expansion. Analytically, we found that all the nonintegrable powers of the loop-energy cancel, supporting the validity of the local renormalization proposed here.

To test the numerical behavior of the locally renormalized amplitude, we started by reparametrizing the energy component of the loop momenta. Following the notation of Sec. III, we used polar coordinates to implement the transformation,

$$
\left(\xi_{1}, \xi_{2}\right) \rightarrow(r \cos \alpha, r \sin \alpha),
$$

with $r \in[0, \infty)$ and $\alpha \in[0, \pi / 2]$. In this way, the highenergy limit corresponds to $r \rightarrow \infty$, whilst the angle $\alpha$ allows to control the overlapping singularities associated to $\ell_{1}$ and $\ell_{2}$. Thus, the amplitude and the double UV counterterm can be generally written as

$$
\begin{aligned}
\mathcal{A}_{H \rightarrow g g}^{(2)} & =\int_{0}^{\infty} d r r \int_{0}^{\pi / 2} d \alpha \int d \Omega f^{(2)}(r, \alpha, \Omega), \\
\mathcal{A}_{\mathrm{UV}^{2}}^{(2)} & =\int_{0}^{\infty} d r r \int_{0}^{\pi / 2} d \alpha \int d \Omega f_{12, \mathrm{UV}^{2}}^{(2)}(r, \alpha, \Omega),
\end{aligned}
$$

where $\Omega$ denotes all the angular variables describing the loop momenta. In Fig. 6, we present a graphical representation of the integrand functions $f^{(2)}$ and $f_{12, \mathrm{UV}^{2}}^{(2)}$ for arbitrary fixed values of the angular variables and the helicity configuration ++ . In these plots, $\delta A$ corresponds to the integrands of Eq. (28), whilst

$$
\delta A_{\mathrm{REN}}=r\left(f^{(2)}-f_{12, \mathrm{UV}^{2}}^{(2)}\right),
$$

i.e., the integrand after subtracting the UV counterterm. Also, we set $m_{H}=125 \mathrm{GeV}, m_{t}=175 \mathrm{GeV}$, and $m_{Z}=91 \mathrm{GeV}$, together with $\mu_{\mathrm{UV}}=m_{H}$ as the renormalization scale. In Fig. 6(a), we appreciate that the amplitude and the counterterm exhibit the same behavior in the
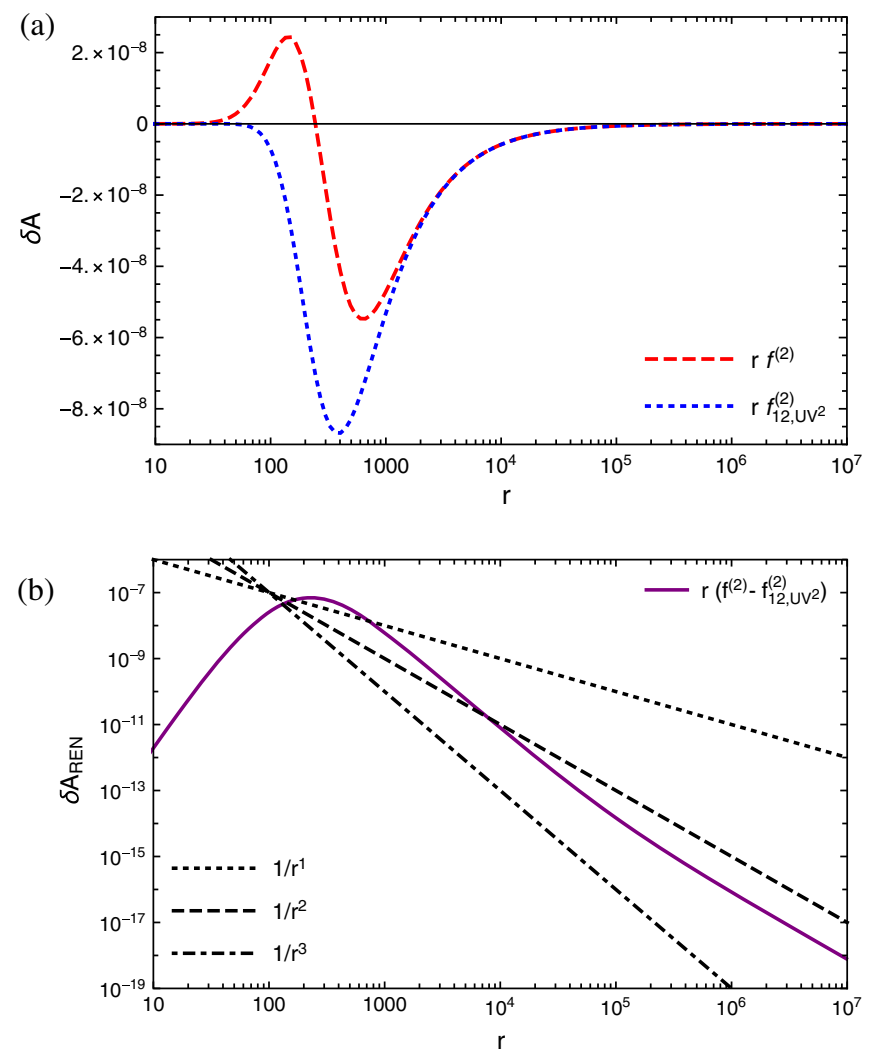

FIG. 6. Integrand-level study of the local cancellation of UV singularities. (a) The UV counterterm (dotted) exactly reproduces the high-energy behavior of the amplitude (dashed). (b) The dotted line scales like $1 / r$ (nonintegrable function); the dashed one corresponds to $1 / r^{2}$ (integrable function). The locally renormalized amplitude (solid line) decreases faster than $1 / r^{2}$ for high energies.

high-energy limit. Additionally, in Fig. 6(b), we notice that the asymptotic limit satisfies

$$
\mid r\left(f^{(2)}-f_{12, \mathrm{UV}^{2}}^{(2)} \mid \leq 1 / r^{\nu} \quad \text { for } r \rightarrow \infty,\right.
$$

with $\nu>1$ since the renormalized integrand (represented by the solid line) decreases faster than $1 / r^{2}$ (dashed line). This constitutes a numerical proof of the convergence of the integral in the UV region. Furthermore, the corresponding analytic expressions for the renormalized amplitude, before integration, are available from the authors upon request.

\section{CONCLUSIONS AND FUTURE DIRECTIONS}

In this paper, we have presented an efficient numerical implementation of helicity amplitudes in the LTD representation. To achieve this, we exploited the prominent fact that LTD changes the loop integration domain from a Minkowski to an Euclidean space. This leads to important numerical simplifications in the integrand-level representation of scattering amplitudes and loop integrals due to the manifest cancellation of unphysical or noncausal 
singularities $[13,14]$. Simultaneously, the application of the helicity formalism leads to expressions depending on a minimal set of kinematical variables, which contributes to speed up the numerical calculation.

We have focused in this paper on helicity amplitudes for physical scattering processes which, although they are UV and IR finite, cannot be calculated by traditional methods naively in four space-time dimensions because they still exhibit a local UV singular behavior. As causal threshold singularities of loop integrals have already been considered in Refs. [65,66], we avoided their presence by choosing proper external kinematics, thus focusing our attention in the local UV counterterms to achieve proper integrability in the high-energy region directly in four space-time dimensions.

The computational framework developed was successfully applied to benchmark helicity amplitudes at one loop. Numerical results were compared to explicit analytical expressions, and we found complete agreement. Additionally, we applied the local renormalization procedure at two loops introduced in Ref. [36] to obtain an integrable four-dimensional representation of the $H \rightarrow g g$ helicity amplitudes with internal top quarks and $Z$ bosons.

This work constitutes an important step towards the automation of a LTD-based framework to compute physical observables in a fully numerical approach [67]. We expect to acquire a better understanding of the local UV renormalization at higher orders and how to increase its efficiency.

\section{ACKNOWLEDGMENTS}

We would like to thank Simon Badger, Pierpaolo Mastrolia, Tiziano Peraro, and Amedeo Primo for illuminating discussions. This work is supported by the Spanish Government (MCIN/AEI/10.13039/501100011033) Grant No. PID2020-114473 GB-I00, Generalitat Valenciana (Grant No. PROMETEO/2021/071), Consejo Superior de Investigaciones Científicas (Grant No. PIE-201750E021), and the COST Action CA16201 PARTICLEFACE. W. J. T. acknowledges support from the Spanish Government (Grant No. FJCI-2017-32128). The Feynman diagrams depicted in this paper are generated using FEYNARTS [68].

\section{APPENDIX A: MOMENTUM TWISTOR PARAMETRIZATION}

For the sake of simplicity, we remark the main features of the twistor variables. Besides, for an exhaustive study of them, we refer the reader to Refs. $[29,69,70]$.

\section{Little group scaling}

Let us remark the little group scaling, a group of transformations that leaves the momentum of an on-shell particle invariant. Hence, the spinors $|i\rangle$ and $\mid i]$ can be rescaled according to

$$
\left.|i\rangle \rightarrow t|i\rangle, \quad \mid i] \rightarrow t^{-1} \mid i\right] .
$$

This transformation turns out to be very interesting at the amplitude level. This is because amplitudes with massless particles can always be written in terms of spinorial products. Then, we have that:

(i) scalar particles do not scale,

(ii) fermions with spin $1 / 2$ scale as $t^{-2 h}$ for $h= \pm \frac{1}{2}$,

(iii) polarization vectors with spin 1 scale as $t^{-2 h}$ for $h= \pm 1$.

This implies that an $\mathrm{N}$-point amplitude, after one of the massless particles is rescaled according to Eq. (A1), can be expressed as,

$$
\begin{aligned}
& \left.\left.\mathcal{A}_{N}\left(\{|1\rangle, \mid 1], h_{1}\right\}, \ldots,\left\{t_{i}|i\rangle, t_{i}^{-1} \mid i\right], h_{i}\right\}, \cdots\right) \\
& \left.\left.\quad=t_{i}^{-2 h_{i}} \mathcal{A}_{N}\left(\{|1\rangle, \mid 1], h_{1}\right\}, \ldots,\left\{t_{i}|i\rangle, t_{i}^{-1} \mid i\right], h_{i}\right\}, \cdots\right),
\end{aligned}
$$

with $h_{i}$ the helicity of the particle $i$.

\section{Momentum twistor variables}

The momentum conservation rule implies that the vectors representing the different momenta close into a contour, which can be defined by the edges or by the cusps. The former is the usual representation, $p_{1}+p_{2}+$ $\cdots+p_{N}=0$, whereas, the latter correspond to locate a point $y_{i}^{\mu}$ in a dual space. In fact, these points can be expressed in terms of momentum vectors,

$$
p_{i}^{\alpha}=\left(y_{i}-y_{i+1}\right)^{\alpha} .
$$

These dual variables satisfy momentum conservation after imposing a periodicity relation, namely $y_{N+1}=y_{1}$. For the sake of simplicity, we take into account the ordering of the external particles. Hence, we define

$$
y_{i j}^{\alpha}=\left(y_{i}-y_{j}\right)^{\alpha}=\left(p_{i}+p_{i+1}+\cdots+p_{j-1}\right)^{\alpha} .
$$

Furthermore, because all the particles are massless (i.e., $p_{i}^{2}=0$ ), we write the Dirac equation in terms of holomorphic spinors,

$$
\not p_{i}|i\rangle=\left(\not_{i}-y_{i+1}\right)|i\rangle=0,
$$

and we define a new variable $\left.\mid \mu_{i}\right]$, according to

$$
\left.\mid \mu_{i}\right]=y_{i}|i\rangle=y_{i+1}|i\rangle \text {. }
$$

With these two independent variables, $|i\rangle$ and $\left.\mid \mu_{i}\right]$, we build a new four-component spinor variable $Z_{i}$, usually called momentum twistor. Nevertheless, the antiholomorphic spinors $\mid i]$ can be written as 


$$
\left[i \mid=\frac{\langle i+1 i\rangle\left[\mu_{i-1} \mid+\langle i i-1\rangle\left[\mu_{i+1} \mid+\langle i-1 i-1\rangle\left[\mu_{i} \mid\right.\right.\right.}{\langle i-1 i\rangle\langle i i+1\rangle}\right.
$$

due to the Gordon identity. Given $n$ momentum twistors, denoted $\left(Z_{1}, Z_{2}, \ldots, Z_{n}\right)$, they must fulfil Poincaré and $\mathrm{U}(1)$ symmetries, besides satisfying momentum conservation and on-shellness. These symmetries allow us to express any $n$-point massless amplitude in terms for $3 N-10$ variables, which is the minimal quantity required [71].

Since we are interested in parametrizing the external momenta in terms of the minimal set of variables, we follow the representation used in Ref. [29]. In particular, for a four-point kinematics, we have

$$
Z=\left(\begin{array}{llll}
|1\rangle & |2\rangle & |3\rangle & |4\rangle \\
\left.\mid \mu_{1}\right] & \left.\mid \mu_{2}\right] & \left.\mid \mu_{3}\right] & \left.\mid \mu_{4}\right]
\end{array}\right)=\left(\begin{array}{cccc}
1 & 0 & \frac{1}{z_{1}} & \frac{1}{z_{1}}+\frac{1}{z_{2}} \\
0 & 1 & 1 & 1 \\
0 & 0 & -1 & -1 \\
0 & 0 & 0 & 1
\end{array}\right),
$$

where we can relate $z_{1}$ and $z_{2}$ to the kinematic invariants according to

$$
z_{1}=s_{12}, \quad z_{2}=\frac{s_{14}}{s_{12}} .
$$

Likewise, we obtain a particular generalization for $N \geq 5$,

$$
Z=\left(\begin{array}{cccccccc}
1 & 0 & f_{1} & f_{2} & f_{3} & \cdots & f_{N-3} & f_{N-2} \\
0 & 1 & 1 & 1 & 1 & \cdots & 1 & 1 \\
0 & 0 & 0 & \frac{z_{N-1}}{z_{2}} & z_{N} & \cdots & z_{2 N-6} & 1 \\
0 & 0 & 1 & 1 & z_{2 N-5} & \cdots & z_{3 N-11} & 1-\frac{z_{3 N-10}}{z_{N-1}}
\end{array}\right),
$$

with

$$
f_{i}=\sum_{k=1}^{i} \frac{1}{\prod_{l=1}^{k} z_{l}}
$$

and

$$
z_{i}= \begin{cases}s_{12} & i=1 \\ -\frac{\langle i i+1\rangle\langle i+21\rangle}{\langle 1 i\rangle\langle i+1 i+2\rangle} & i=2, \ldots, N-2 \\ \frac{s_{23}}{s_{12}} & i=N-1 \\ \sum_{j=2}^{i-N+4} \frac{\langle i-N+5|j| 2]}{[12]\langle 1 i-N+5\rangle} & i=N, \ldots, 2 N-6 \\ \sum_{j=2}^{i-2 N+9} \frac{\langle 1|(2+3) j| i-2 N+10\rangle}{s_{23}\langle 1 i-2 N+10\rangle} & i=2 N-5, \ldots, 3 N-11 \\ \frac{s_{123}}{s_{12}} & i=3 N-10 .\end{cases}
$$

We remark that with this configuration of external momenta, we drop the physical phase of the amplitude, namely, the information that accounts for parity invariance. However, it can be straightforwardly restored by using the prefactor

$$
\left(\frac{\langle 13\rangle}{[12]\langle 23\rangle}\right)^{-h_{1}} \prod_{i=2}^{n}\left(\frac{\langle 1 i\rangle^{2}[12]\langle 23\rangle}{\langle 13\rangle}\right)^{-h_{i}},
$$

where $h_{i}$ are the helicities of the external massless momenta.

\section{APPENDIX B: EXTERNAL MOMENTA}

In this Appendix we give the external momenta in terms of the kinematic scales shown in Secs. V and VI.

$$
\text { 1. } H \rightarrow \gamma \gamma \text { and } H \rightarrow g g
$$

We focus on the process

$$
H\left(-p_{3}\right) \rightarrow g\left(p_{1}\right)+g\left(p_{2}\right),
$$

with the kinematics,

$$
\begin{aligned}
p_{1}^{\mu} & =\frac{1}{2}\{-1,1, \imath,-1\}, \\
p_{2}^{\mu} & =\frac{s_{12}}{2}\{0,-1, l, 0\}, \\
\varepsilon_{+}^{\mu}\left(p_{1}\right) & =\frac{1}{\sqrt{2}}\{1,-1, l,-1\}, \\
\varepsilon_{-}^{\mu}\left(p_{1}\right) & =\frac{1}{\sqrt{2}}\{-1,0,0,-1\}, \\
\varepsilon_{+}^{\mu}\left(p_{2}\right) & =\frac{s_{12}}{\sqrt{2}}\{1,0,0,1\}, \\
\varepsilon_{-}^{\mu}\left(p_{2}\right) & =\frac{1}{\sqrt{2} s_{12}}\{-1,1,-l, 1\} .
\end{aligned}
$$

$$
\text { 2. } \gamma \gamma \rightarrow \gamma \gamma
$$

We consider the light-by-light scattering,

$$
\gamma\left(-p_{1}\right) \gamma\left(-p_{2}\right) \rightarrow \gamma\left(p_{3}\right) \gamma\left(p_{4}\right),
$$

with the kinematics,

$$
\begin{aligned}
p_{1}^{\mu} & =\frac{1}{2}\{-1,1, l,-1\}, \\
p_{2}^{\mu} & =\frac{s}{2}\{0,-1, l, 0\}, \\
p_{3}^{\mu} & =\frac{1}{2}\{s t+1, s+t, l(t-s), 1-s t\}, \\
\varepsilon_{+}^{\mu}\left(p_{1}\right) & =\frac{1}{\sqrt{2}}\{1,-1, l,-1\}, \\
\varepsilon_{-}^{\mu}\left(p_{1}\right) & =\frac{1}{\sqrt{2}}\{-1,0,0,-1\},
\end{aligned}
$$




$$
\begin{aligned}
\varepsilon_{+}^{\mu}\left(p_{2}\right)= & \frac{s}{\sqrt{2}}\{1,0,0,1\}, \\
\varepsilon_{-}^{\mu}\left(p_{2}\right)= & \frac{1}{\sqrt{2} s}\{-1,1,-l, 1\} . \\
\varepsilon_{+}^{\mu}\left(p_{3}\right)= & \frac{s}{\sqrt{2}}\{-1,-t,-l t,-s\}, \\
\varepsilon_{-}^{\mu}\left(p_{3}\right)= & \frac{1}{\sqrt{2} s^{2}(t+1)} \\
& \times\{s-1,-(s-1), t(s+1),-(s+1)\}, \\
\varepsilon_{+}^{\mu}\left(p_{4}\right)= & \frac{s t}{\sqrt{2}}\{0,1, t, 0\}, \\
\varepsilon_{-}^{\mu}\left(p_{4}\right)= & \frac{1}{\sqrt{2} s^{2} t^{2}}\{-s t+t+1,(s-1) t-1, \\
& -\imath(s t+t+1), s t+t+1\} . \\
& \mathbf{3 .} \boldsymbol{H} \rightarrow \boldsymbol{g g g}
\end{aligned}
$$

\section{3. $H \rightarrow g g g$}

We consider the Higgs decay into thee gluons,

$$
H \rightarrow g\left(p_{2}\right) g\left(p_{3}\right) g\left(p_{4}\right)
$$

with

$$
\begin{aligned}
p_{1}^{\mu} & =\frac{1}{2}\left\{\frac{s_{12}+s_{13}}{s_{23}}, 1, l, \frac{s_{12}+s_{13}}{s_{23}}\right\}, \\
p_{2}^{\mu}= & \frac{s_{12}}{2}\{0,-1, l, 0\}, \\
p_{3}^{\mu}= & \frac{1}{2}\left\{s_{23}+1, \frac{s_{23}}{s_{12}}+s_{12}, l\left(\frac{s_{23}}{s_{12}}-s_{12}\right), 1-s_{23}\right\}, \\
\varepsilon_{+}^{\mu}\left(p_{1}\right)= & \frac{1}{\sqrt{2}}\{1,-1, l,-1\}, \\
\varepsilon_{-}^{\mu}\left(p_{1}\right)= & \frac{1}{\sqrt{2}}\{-1,0,0,-1\}, \\
\varepsilon_{+}^{\mu}\left(p_{2}\right)= & \frac{s_{12}}{\sqrt{2}}\{1,0,0,1\}, \\
\varepsilon_{-}^{\mu}\left(p_{2}\right)= & \frac{1}{\sqrt{2} s_{12}}\{-1,1,-l, 1\} . \\
\varepsilon_{+}^{\mu}\left(p_{3}\right)= & \frac{1}{\sqrt{2}}\left\{-s_{12},-s_{23},-l s_{23},-s_{12}\right\} \\
\varepsilon_{-}^{\mu}\left(p_{3}\right)= & \frac{1}{\sqrt{2} s_{12} s_{13} s_{23}} \\
& \times\left\{-\left(s_{12} s_{23}+s_{12}+s_{13}\right),-\left(s_{12}\left(s_{12}+s_{13}\right)+s_{23}\right),\right. \\
& \left.+\imath\left(s_{12}\left(s_{12}+s_{13}\right)-s_{23}\right),-\left(s_{12}+s_{13}-s_{12} s_{23}\right)\right\} .
\end{aligned}
$$

[1] L. J. Dixon, in Theoretical Advanced Study Institute in Elementary Particle Physics (TASI 95): QCD and Beyond (1996), pp. 539-584 [arXiv:hep-ph/9601359].

[2] Z. Bern and D. A. Kosower, Nucl. Phys. B362, 389 (1991).

[3] R. Kleiss and H. Kuijf, Nucl. Phys. B312, 616 (1989).

[4] Z. Bern, J. J. M. Carrasco, and H. Johansson, Phys. Rev. D 78, 085011 (2008).

[5] C. Gnendiger et al., Eur. Phys. J. C 77, 471 (2017).

[6] W. J. Torres Bobadilla et al., Eur. Phys. J. C 81, 250 (2021).

[7] For a detailed review on analytic and semi-numerical techniques, we refer the reader to Ref. [8].

[8] G. Heinrich, Phys. Rep. 922, 1 (2021).

[9] S. Catani, T. Gleisberg, F. Krauss, G. Rodrigo, and J.-C. Winter, J. High Energy Phys. 09 (2008) 065.

[10] I. Bierenbaum, S. Catani, P. Draggiotis, and G. Rodrigo, J. High Energy Phys. 10 (2010) 073.

[11] I. Bierenbaum, S. Buchta, P. Draggiotis, I. Malamos, and G. Rodrigo, J. High Energy Phys. 03 (2013) 025.

[12] J. Plenter and G. Rodrigo, Eur. Phys. J. C 81, 320 (2021).

[13] J. J. Aguilera-Verdugo, F. Driencourt-Mangin, J. Plenter, S. Ramírez-Uribe, G. Rodrigo, G. F. R. Sborlini, W. J. Torres Bobadilla, and S. Tracz, J. High Energy Phys. 12 (2019) 163.

[14] J. J. Aguilera-Verdugo, F. Driencourt-Mangin, R. J. Hernández-Pinto, J. Plenter, S. Ramirez-Uribe, A. E.
Renteria Olivo, G. Rodrigo, G. F. R. Sborlini, W. J. Torres Bobadilla, and S. Tracz, Phys. Rev. Lett. 124, 211602 (2020).

[15] J. J. Aguilera-Verdugo, R. J. Hernandez-Pinto, G. Rodrigo, G. F. R. Sborlini, and W. J. Torres Bobadilla, J. High Energy Phys. 01 (2021) 069.

[16] S. Ramírez-Uribe, R. J. Hernández-Pinto, G. Rodrigo, G. F. R. Sborlini, and W. J. Torres Bobadilla, J. High Energy Phys. 04 (2021) 129.

[17] J. J. Aguilera-Verdugo, R. J. Hernández-Pinto, G. Rodrigo, G. F. R. Sborlini, and W. J. Torres Bobadilla, J. High Energy Phys. 02 (2021) 112.

[18] J. de Jesús Aguilera-Verdugo et al., Symmetry 13, 1029 (2021).

[19] G. F. R. Sborlini, Phys. Rev. D 104, 036014 (2021).

[20] W. J. Torres Bobadilla, J. High Energy Phys. 04 (2021) 183.

[21] E. T. Tomboulis, J. High Energy Phys. 05 (2017) 148.

[22] R. Runkel, Z. Szőr, J. P. Vesga, and S. Weinzierl, Phys. Rev. Lett. 122, 111603 (2019); 123, 059902(E) (2019).

[23] Z. Capatti, V. Hirschi, D. Kermanschah, and B. Ruijl, Phys. Rev. Lett. 123, 151602 (2019).

[24] Z. Capatti, V. Hirschi, D. Kermanschah, A. Pelloni, and B. Ruijl, arXiv:2009.05509.

[25] Z. Capatti, V. Hirschi, A. Pelloni, and B. Ruijl, J. High Energy Phys. 04 (2021) 104. 
[26] R. M. Prisco and F. Tramontano, J. High Energy Phys. 06 (2021) 089.

[27] A. Hodges, J. High Energy Phys. 05 (2013) 135.

[28] S. Badger, H. Frellesvig, and Y. Zhang, J. High Energy Phys. 12 (2013) 045.

[29] S. Badger, J. Phys. Conf. Ser. 762, 012057 (2016).

[30] F. Driencourt-Mangin, G. Rodrigo, and G. F. R. Sborlini, Eur. Phys. J. C 78, 231 (2018).

[31] S. Becker, C. Reuschle, and S. Weinzierl, J. High Energy Phys. 12 (2010) 013.

[32] S. Becker, C. Reuschle, and S. Weinzierl, J. High Energy Phys. 07 (2012) 090.

[33] R. J. Hernandez-Pinto, G. F. R. Sborlini, and G. Rodrigo, J. High Energy Phys. 02 (2016) 044.

[34] G. F. R. Sborlini, F. Driencourt-Mangin, R. HernandezPinto, and G. Rodrigo, J. High Energy Phys. 08 (2016) 160.

[35] G. F. R. Sborlini, F. Driencourt-Mangin, and G. Rodrigo, J. High Energy Phys. 10 (2016) 162.

[36] F. Driencourt-Mangin, G. Rodrigo, G. F. R. Sborlini, and W. J. Torres Bobadilla, J. High Energy Phys. 02 (2019) 143.

[37] R. P. Feynman, Acta Phys. Pol. 24, 697 (1963).

[38] F. V. Tkachov, Phys. Lett. 100B, 65 (1981).

[39] K. G. Chetyrkin and F. V. Tkachov, Nucl. Phys. B192, 159 (1981).

[40] S. Laporta, Int. J. Mod. Phys. A 15, 5087 (2000).

[41] L. Chen, Eur. Phys. J. C 81, 417 (2021).

[42] T. Peraro and L. Tancredi, J. High Energy Phys. 07 (2019) 114.

[43] G. 't Hooft and M. J. G. Veltman, Nucl. Phys. B44, 189 (1972).

[44] Z. Bern, A. De Freitas, L. J. Dixon, and H. L. Wong, Phys. Rev. D 66, 085002 (2002).

[45] The FDH scheme at one-loop level has been considered in a purely four-dimension formulation in Ref. [46-48].

[46] R. A. Fazio, P. Mastrolia, E. Mirabella, and W. J. Torres Bobadilla, Eur. Phys. J. C 74, 3197 (2014).

[47] P. Mastrolia, A. Primo, U. Schubert, and W. J. Torres Bobadilla, Phys. Lett. B 753, 242 (2016).

[48] A. Primo and W. J. Torres Bobadilla, J. High Energy Phys. 04 (2016) 125.

[49] We closely follow the convention of Ref. [5].
[50] For more details about the construction of the momentum parametrisation, see Appendix A.

[51] R. Mertig, M. Bohm, and A. Denner, Comput. Phys. Commun. 64, 345 (1991).

[52] V. Shtabovenko, R. Mertig, and F. Orellana, Comput. Phys. Commun. 207, 432 (2016).

[53] V. Shtabovenko, Comput. Phys. Commun. 218, 48 (2017).

[54] H. H. Patel, Comput. Phys. Commun. 197, 276 (2015).

[55] G. Ossola, C. G. Papadopoulos, and R. Pittau, Nucl. Phys. B763, 147 (2007).

[56] P. Mastrolia and G. Ossola, J. High Energy Phys. 11 (2011) 014.

[57] S. Badger, H. Frellesvig, and Y. Zhang, J. High Energy Phys. 04 (2012) 055.

[58] Y. Zhang, J. High Energy Phys. 09 (2012) 042.

[59] P. Mastrolia, E. Mirabella, G. Ossola, and T. Peraro, Phys. Lett. B 718, 173 (2012).

[60] P. Mastrolia, E. Mirabella, G. Ossola, and T. Peraro, Phys. Rev. D 87, 085026 (2013).

[61] H. Ita, Phys. Rev. D 94, 116015 (2016).

[62] P. Mastrolia, T. Peraro, and A. Primo, J. High Energy Phys. 08 (2016) 164.

[63] P. Mastrolia, T. Peraro, A. Primo, and W. J. Torres Bobadilla, Proc. Sci., LL2016 (2016) 007 [arXiv:1607.05156].

[64] We attach in the ancilliary files AmplitudeCuts.m and UVCountertermcuts.m the double cuts for the amplitude (diagram by diagram) and the local UV counter-term, respectively.

[65] S. Buchta, Theoretical foundations and applications of the loop-tree duality in quantum field theories, Ph.D. thesis, Valencia Univerity, 2015.

[66] S. Buchta, G. Chachamis, P. Draggiotis, and G. Rodrigo, Eur. Phys. J. C 77, 274 (2017).

[67] W. J. Torres Bobadilla, Eur. Phys. J. C 81, 514 (2021).

[68] T. Hahn, Comput. Phys. Commun. 140, 418 (2001).

[69] T. Peraro, J. High Energy Phys. 12 (2016) 030.

[70] W. J. Torres Bobadilla, Generalised unitarity, integrand decomposition, and hidden properties of QCD scattering amplitudes in dimensional regularisation, Ph.D. thesis, Padua Univeristy, 2017.

[71] For an extensive review of the derivation of the momentum twistors, we refer the reader to Refs. [29]. 